\title{
Comparison between loop-tip guidewire-assisted and conventional endoscopic cannulation in high risk patients
}

Authors

Institutions
Enzo Masci ${ }^{1}$, Benedetto Mangiavillano ${ }^{1}$, Carmelo Luigiano ${ }^{2}$, Alessandra Bizzotto ${ }^{1}$, Eugenio Limido ${ }^{3}$, Paolo Cantù ${ }^{4}$, Gianpiero Manes ${ }^{5}$, Paolo Viaggi ${ }^{1}$, Giancarlo Spinzi ${ }^{6}$, Franco Radaelli ${ }^{6}$, Alberto Mariani ${ }^{7}$, Clara Virgilio ${ }^{2}$, Angela Alibrandi ${ }^{8}$, Pier Alberto Testoni ${ }^{7}$

Institutions are listed at the end of article. submitted 7. April 2015 accepted after revision 26. June 2015

\section{Bibliography}

Dol http://dx.doi.org/

10.1055/s-0034-1392879

Published online: 15.9.2015

Endoscopy International Open 2015; 03: E464-E470

(c) Georg Thieme Verlag KG Stuttgart · New York

E-ISSN 2196-9736

\section{Corresponding author}

Enzo Masci, MD

Gastrointestinal Endoscopy Unit

San Paolo University Hospital

University of Milan

Via A. Di Rudinì 8

20142 Milano

Italy

Fax: +39-0184-536870

enzo.masci@fastwebnet.it
Background: The guidewire biliary cannulation (GWC) technique may increase the cannulation rate and decrease the risk for post-endoscopic retrograde cholangiopancreatography (ERCP) pancreatitis. The aim of our multicenter prospective randomized controlled trial was to determine if the use of an atraumatic loop-tip guidewire reduces the rate of post-ERCP pancreatitis (PEP) compared with the standard contrast-assisted cannulation (CC) technique.

Methods: From June 2012 to December 2013, a total of 320 patients who had a naïve papilla and were referred for ERCP were randomly assigned to the GWC group $(n=160)$ or the CC group $(n=$ 160). GWC or CC was randomly used. In cases of failed cannulation in both arms after crossover, biliary access was attempted with alternative

\section{Introduction}

$\nabla$

Acute pancreatitis remains the most frequent and dreaded complication after endoscopic retrograde cholangiopancreatography (ERCP), with rates ranging from $2 \%$ to $40 \%$ in published series [1-3]. Although in most cases post-ERCP pancreatitis (PEP) manifests as a mild, uneventful disease requiring 2 to 3 days of additional hospitalization with an expected full recovery, severe pancreatitis is a devastating occurrence fraught with a significant mortality rate. Several risk factors for ERCP-related complications have been clearly identified, and various strategies have been proposed aimed at reducing the risk for PEP $[4,5]$ Repeated attempts at cannulation and increased pressure within the pancreatic duct (PD) due to contrast injection into the main PD result in trauma to the papilla and are major risk factors for PEP [6]. Other factors shown to increase the risk for PEP include female sex, younger age, and sphincter of Oddi dysfunction.

License terms

()(1) $\Theta \circledast$ costeroids, nonsteroidal anti-inflammatory drugs,
Various pharmacological agents, including corti- techniques (e.g., dual-wire technique, pancreatic duct stenting, precut).

Results: The biliary cannulation rates were $81 \%$ in the GWC group and $73 \%$ in the CC group $(P=$ n.s.). Following crossover, cannulation was successful in $8 \%$ and $11 \%$ of patients in the GWC and CC groups, respectively. With use of an alternative technique, the cannulation rates were $98 \%$ in the GWC group and $96 \%$ in the CC group, respectively. The rates of PEP were $5 \%$ in the GWC group and $12 \%$ in the CC group $(P=0.027)$. The post-interventional complication rates did not differ between the two groups.

Conclusion: GWC with the new wire guide is associated with a lower rate of PEP in comparison with the CC technique.

Clinical trial reference number: NCT01771419

somatostatin analogues, and protease inhibitors, have been investigated; however, the benefit and advisability of their routine use remain uncertain $[7,8]$. As well, various procedural techniques, such as the insertion of pancreatic stents or the use of pure cut electrocautery for sphincterotomy, have been suggested to minimize the development of PEP, but most studies have produced nonconfirmatory data.

Most nonrandomized and randomized studies have compared the effectiveness of guidewireassisted cannulation (GWC) with that of contrast-assisted cannulation (CC) for the prevention of PEP but are restricted to low risk patients, and the results so far are conflicting [9-18].

The rationale for common bile duct (CBD) cannulation with the guidewire-assisted technique is that it is easier to pass a hydrophilic guidewire with a small diameter through the small opening of the CBD than a larger catheter or sphincterotome. However, this cannulation technique carries a risk for false passage, intramural dissection, perforation, and PD injury [6]. 
Recently, a new wire guide with an atraumatic loop tip has been marketed (Fusion LoopTip Wire Guide; Cook Medical, Bloomington, Indiana, USA). The design of the device may reduce injury to the pancreatic and bile ducts during cannulation attempts.

The aim of this study was to assess if this atraumatic loop-tip guidewire reduces the occurrence of PEP (the primary end point). As a secondary end point, we evaluated whether the use of a loop-tip guidewire increases the rate of $\mathrm{CBD}$ cannulation in patients at higher risk for developing PEP in comparison with the $\mathrm{CC}$ technique.

\section{Patients and methods}

$\nabla$

Between June 2012 to December 2013, a prospective study was carried out at eight Italian tertiary referral endoscopy centers. The study protocol was conducted in accordance with the principles of good clinical practice and the Declaration of Helsinki (clinical trials reference number NCT01771419), and the institutional review boards of the hospitals approved the study protocol. All patients provided written informed consent to take part in the study. Eleven endoscopists with experience of more than 150 ERCPs per year conducted the procedures (E.M., B.M., C.L., E.L., P.C., G.M., P.V., F.R., A.M., C.V., and P.A.T.). All of the endoscopists used both methods (GWC and CC) to cannulate the CBD.

\section{Fusion loop-tip guidewire}

The loop-tip wire was developed to assist in cannulating the bile and pancreatic ducts and in bridging difficult strictures during ERCP. The wire has a closed distal loop that causes less trauma to the pancreaticobiliary ductal system during access. The coil is radiopaque with optimal fluoroscopic visibility. Like other types of wire, the loop-tip guidewire has spiral markings extending to the distal end, which provide an endoscopic view of its movement during cannulation. The core of the guidewire is made of nitinol for kink resistance and shape retention.

\section{Eligibility criteria}

All consecutive patients 18 years of age or older were considered for inclusion if they met the following criteria: were able to comply with the study procedures and provide written informed consent; had received a diagnosis of a pancreaticobiliary disease and been referred for ERCP; had an intact papilla and one or more risk factors for the development of PEP (CBD diameter $<10 \mathrm{~mm}$, previous episode of acute pancreatitis or recurrent acute pancreatitis, sphincter of Oddi dysfunction type 1 , female sex). The diagnosis of a pancreaticobiliary disorder was based on clinical and laboratory data and findings on computed tomography, magnetic resonance imaging, and/or endoscopic ultrasound.

Exclusion criteria were the following: age younger than 18 years; previous sphincterotomy; previous endoscopic papillary balloon dilation; plastic or metal biliary stent placement; esophageal or gastroduodenal stenting; pancreatic or ampullary carcinoma; prior Billroth II gastrectomy; intradiverticular papilla; duodenal stenosis; contraindications to endoscopy; acute illness (hypotension [blood pressure $<90 \mathrm{mmHg}$ ], hypoxemia [ $\mathrm{SaO}_{2}<95 \%$ ], hemodynamic instability); coagulopathy (platelet count $<50 \times 10^{9} / \mathrm{L}$, international normalized ratio $>1.5$ ); inability or refusal to give written informed consent.

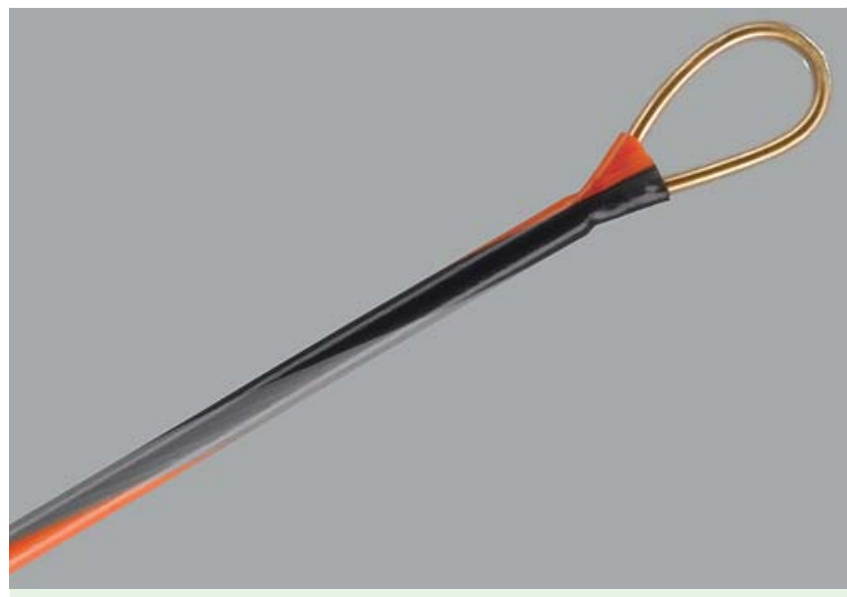

Fig. 1 Loop-tip wire.

\section{Randomization}

Before ERCP, enrolled patients were randomly allocated to one of two groups (GWC group or CC group) on the basis of a sealed envelope method used by a physician who was not involved in performing ERCP or in the critical care of the patients. Data from consecutive patients were collected in a prospective manner through a standardized case report form and centralized at a coordinating center for analysis and interpretation.

\section{Study protocol}

ERCP procedures were conducted by experienced therapeutic endoscopists using a therapeutic video duodenoscope.

Guidewire cannulation group: In the GWC group, the sphincterotome was preloaded with a 0.035 -in loop-tip guidewire ( $\bullet$ Fig. 1). The sphincterotome was oriented from the 11- to the 12-o'clock position on the papilla and bent to align it correctly with the bile duct axis. After a minimal insertion $(2-3 \mathrm{~mm})$ of the sphincterotome across the ampulla, the guidewire was carefully advanced through the CBD under fluoroscopy until it was seen entering the bile duct ( $\bullet$ Fig. 2). In cases of PD cannulation, the guidewire was withdrawn, and attempts were made to redirect it toward the $\mathrm{CBD}$. Such attempts were continued for no longer than 5 minutes or up to five unintentional cannulations of the PD. If biliary cannulation was achieved through guidewire insertion, contrast medium was injected, whereas in a case of failure, the patient was crossed over to the CC technique for a time limit of 5 minutes or for a maximum of three repeated, unintentional contrast injections or cannulations of the main PD.

Contrast-assisted cannulation group: In the CC group, the sphincterotome was used to cannulate the CBD directly, with its position adjusted to the correct axis for bile duct cannulation ( $\bullet$ Fig. 3 ). If direct cannulation was not achieved, contrast opacifications were performed to visualize the $\mathrm{CBD}$, and the sphincterotome was deeply reinserted under cholangiographic guidance. Such attempts were continued for 5 minutes or for a maximum of three unintentional PD opacifications or cannulations. If the procedure did not succeed with the CC method at the first attempt, patients were crossed over to the GWC technique with the loop-tip guidewire, and the endoscopist attempted to cannulate for no longer than 5 minutes or five accidental main PD cannulations.

The number of chances of main PD cannulation for the GWC group (five) and for the CC group (three) were chosen only for the study protocol. In cases of failed cannulation with both techniques, alternative techniques to access the biliary tree were 


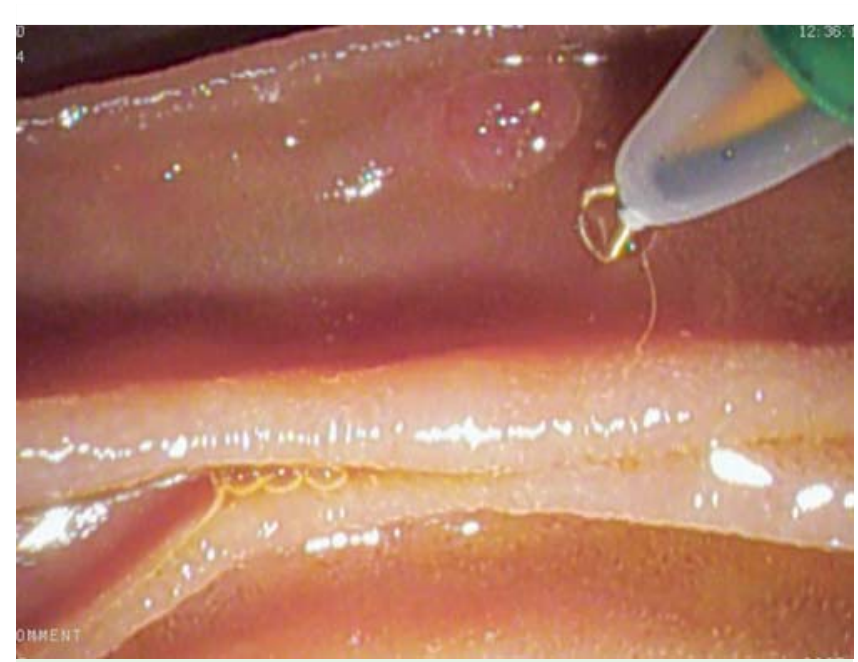

Fig. 2 Loop-tip wire outside the sphincterotome before cannulation of the common bile duct.

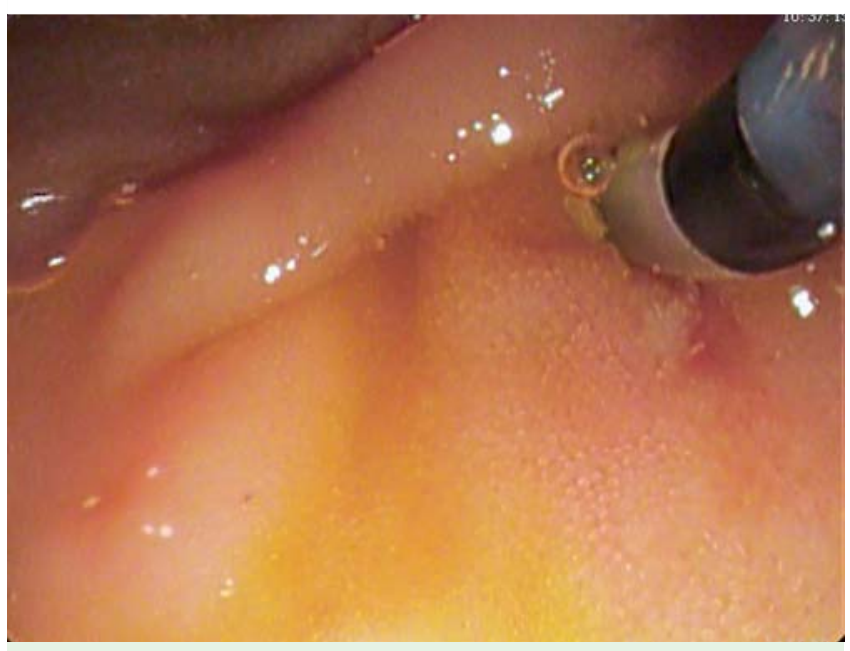

Fig. 3 Contrast-assisted cannulation without use of a guidewire.

adopted, such as dual-wire technique, PD stenting, rendez-vous, or precut, according to the endoscopist's experience.

None of the patients received nonsteroidal anti-inflammatory drugs or a prophylactic pancreatic stent.

\section{Definition of events}

Successful deep biliary cannulation was defined as free and deep instrumentation of the biliary tree. The definition and grading of complications of ERCP and therapy were based on consensus guidelines [1].

A diagnosis of PEP was established in the presence of typical abdominal pain associated with serum amylase elevations more than threefold above the upper limit of normal on day 1 after the procedure. The severity of pancreatitis was graded as mild (2 - 3 days of hospitalization), moderate (4-10 days of hospitalization), or severe ( $\geq 10$ days of hospitalization or a requirement for admission to the intensive care unit and/or endoscopic/radiological percutaneous drainage or surgical intervention).

The severity of bleeding was defined as mild (endoscopic and clinical evidence of bleeding with a hemoglobin drop of $<3 \mathrm{~g} / \mathrm{dL}$ and requiring no blood transfusion), moderate (requiring $<4$ units of blood transfusion and no angiographic intervention/surgery), or severe (requiring $\geq 4$ units of blood transfusion or angiographic intervention/surgery).

The severity of perforation was defined as mild (uncertain or very slight leak of contrast and conservative medical management for $\leq 3$ days), moderate (any definite perforation requiring conservative medical treatment for 4-10 days), or severe (any definite perforation requiring medical treatment for $>10$ days or percutaneous/surgical intervention).

\section{Outcome measures}

Primary outcomes measures were the rate of successful biliary cannulation and incidence of PEP. Secondary outcome measures were the incidence of bleeding, incidence of perforation, number of attempts at biliary cannulation, and number of unintentional PD cannulations/opacifications.

\section{Statistical analysis}

Prior power calculations assumed a PEP rate of $15 \%$ based on the CC technique. Assuming a reduction in PEP of $75 \%$, a sample size of 160 patients per arm was needed to achieve $80 \%$ power with use of a $\mathrm{X}^{2}$ test of proportions at two-sided $5 \%$ significance level. Continuous data were described as mean, standard deviation, and range according to distribution, and categorical data were measured as numbers and percentages. Comparison between groups was assessed by using the Mann-Whitney $U$ test for quantitative variables, and by using the $\mathrm{X}^{2}$ test for qualitative variables. Correlation between variables was evaluated with Spearman's correlation coefficient.

A logistic regression analysis was performed to identify risk factors for the development of complications. A $P$ value below .05 was considered statistically significant. All analyses were performed with SPSS for Windows 11.0 (2001) and Methodologica S.R.L. (2001) software. Data were analyzed on a "per-protocol" basis.

\section{Results}

$\nabla$

During the study period, a total of 320 patients (142 men, 178 women, mean age 65 years) were enrolled and randomized in equal numbers to undergo ERCP with either the GWC technique (GWC group, $n=160$ ) or the CC technique (CC group, $n=160$ ). The patient demographics and baseline data are summarized in - Table 1.

There were no significant differences between the two groups in regard to age, sex, risk factors associated with the development of PEP, and serum amylase level before ERCP, and there was no significant difference between them in regard to underlying pancreaticobiliary disorder $(\square$ Table 1$)$. The most common reason for referral in both groups was CBD stones.

The study results are reported in 0 Table 2. Primary CBD cannulation proved successful in 130 of 160 patients in the GWC group ( $81 \%$ ) and 117 of 160 patients in the CC group ( $73 \%$; $P=$ n. s.). The mean number ( \pm standard deviation $[S D]$ ) of attempts at biliary cannulation was $3 \pm 2$ in the GWC group and $4 \pm 3$ in the CC group $(P=.002)$, and the mean number $( \pm S D)$ of unintentional pancreatic duct guidewire cannulations/opacifications by contrast injection during the attempts was $0.5 \pm 1.1$ in the GWC group and $0.9 \pm 1.3$ in the CC group $(P<.001)$.

Primary CBD cannulation within the randomly assigned technique failed in 73 patients (30 in the GWC group, 43 in the CC 


\begin{tabular}{|c|c|c|c|c|}
\hline & $\begin{array}{l}\text { All patients } \\
(n=320)\end{array}$ & $\begin{array}{l}\text { Guidewire group } \\
(n=160)\end{array}$ & $\begin{array}{l}\text { Contrast group } \\
(n=160)\end{array}$ & $P$ value \\
\hline Age, mean $\pm S D, y$ & $65 \pm 16$ & $64 \pm 15$ & $65 \pm 17$ & n.s. \\
\hline Sex, male/female, $\mathrm{n}$ & $142 / 178$ & $70 / 90$ & $72 / 88$ & n.s. \\
\hline Previous acute or recurrent pancreatitis, $n$ (\%) & $71(22)$ & $37(23)$ & $34(21)$ & n.s. \\
\hline Age $\leq 40 y, n(\%)$ & $29(9)$ & $13(8)$ & $16(10)$ & n.s. \\
\hline Common bile duct $\leq 1 \mathrm{~cm}, \mathrm{n}(\%)$ & $268(84)$ & $131(82)$ & $137(85)$ & n.s. \\
\hline \multicolumn{5}{|l|}{ Indication } \\
\hline Common bile duct stones, $\mathrm{n}(\%)$ & $277(87)$ & $141(88)$ & $136(85)$ & n.s. \\
\hline Intrahepatic lithiasis, n (\%) & $3(0.9)$ & $1(0.6)$ & $2(1.3)$ & n.s. \\
\hline Sphincter of Oddi dysfunction, $\mathrm{n}(\%)$ & $22(7)$ & $10(6)$ & $12(7.5)$ & n.s. \\
\hline Cholangitis, $\mathrm{n}(\%)$ & $34(11)$ & $16(10)$ & $18(11)$ & n.s. \\
\hline Bile leak, $\mathrm{n}(\%)$ & $10(3)$ & $5(3)$ & $5(3)$ & n.s. \\
\hline Preprocedural serum amylase level, mean \pm SD, U/L & $186 \pm 475$ & $248 \pm 608$ & $124 \pm 278$ & n.s. \\
\hline
\end{tabular}

SD, standard deviation; n. s., not significant.

Table 2 Results of the comparison of the two techniques.

\begin{tabular}{|c|c|c|c|}
\hline & $\begin{array}{l}\text { Guidewire group } \\
(n=160)\end{array}$ & $\begin{array}{l}\text { Contrast group } \\
(n=160)\end{array}$ & $P$ value \\
\hline Successful primary common bile duct cannulation, $\mathrm{n}(\%)$ & $130(81)$ & $117(73)$ & n.s. \\
\hline Attempts at bile duct cannulation, mean $\pm S D$ & $3 \pm 2$ & $4 \pm 3$ & .002 \\
\hline Pancreatic opacifications/cannulations, mean $\pm S D$ & $0.5 \pm 1.1$ & $0.9 \pm 1.3$ & $<.001$ \\
\hline Number of crossovers (\%) & $30(19)$ & $43(27)$ & n.s. \\
\hline Number of attempts at secondary bile duct cannulation, mean \pm SD & $1 \pm 2$ & $1 \pm 3$ & n.s. \\
\hline Number of pancreatic opacifications/cannulations, mean \pm SD & $0.2 \pm 0.7$ & $0.2 \pm 07$ & n.s. \\
\hline Number of common bile duct cannulations successful at crossovers (\%) & $13(8)$ & $18(11)$ & n.s. \\
\hline Alternative cannulation technique, $\mathrm{n}(\%)$ & $15(9.3)$ & $25(15.6)$ & n.s. \\
\hline Precut & 8 & 12 & n.s. \\
\hline Rendez-vous & - & 1 & n.s. \\
\hline Double-guidewire & 7 & 12 & n.s. \\
\hline Successful final bile duct cannulation, $n$ (\%) & $157(98)$ & $153(96)$ & n.s. \\
\hline Biliary sphincterotomy, n (\%) & $154(96)$ & $152(95)$ & n.s. \\
\hline Pancreatic sphincterotomy, n (\%) & $2(1.3)$ & $3(1.9)$ & n.s. \\
\hline Common bile duct stones extraction, $\mathrm{n}(\%)$ & $134(84)$ & $120(75)$ & n.s. \\
\hline Intrahepatic stones extraction, $\mathrm{n}(\%)$ & $1(0.6)$ & $3(1.9)$ & n.s. \\
\hline Plastic biliary stenting, $\mathrm{n}(\%)$ & $14(9)$ & $14(9)$ & n.s. \\
\hline Metal biliary stenting, $\mathrm{n}(\%)$ & $1(0.6)$ & $1(0.6)$ & n.s. \\
\hline Nasobiliary drainage, $n(\%)$ & $14(9)$ & $13(8)$ & n.s. \\
\hline Serum amylase level at $24 \mathrm{~h}$, mean $\pm S D, U / L$ & $283 \pm 420$ & $404 \pm 749$ & n.s. \\
\hline Complications, n (\%) & $11(6.9)$ & $22(13.7)$ & n.s. \\
\hline Pancreatitis & $8(5)$ & $19(11.9)$ & .027 \\
\hline Bleeding & 2 & 3 & n.s. \\
\hline Others & 1 & - & n.s. \\
\hline
\end{tabular}

n. s., not significant; SD, standard deviation.

group) in the allotted time. CBD cannulation with the CC technique after crossover was realized in 13 of the 30 patients (43\%) in whom primary cannulation with the GWC technique had been unsuccessful. After crossover, CBD cannulation was achieved with the GWC technique in 18 of the 43 patients (42\%) in the CC group in whom primary cannulation had failed.

At the crossover step, the mean number $( \pm S D)$ of attempts to achieve cannulation (GWC group $1 \pm 2$ vs. CC group $1 \pm 3$ ) and the mean number $( \pm S D)$ of unintentional pancreatic duct opacifications/cannulations (GWC group $0.2 \pm 0.7$ vs. CC group $0.2 \pm 7$ ) were similar in the two groups.

When an alternative rescue cannulation technique was used, the overall successful cannulation rate was $98 \%$ in the GWC group and $96 \%$ in the CC group.
Rates of overall procedure-related complications (PEP, hyperamylasemia, bleeding) did not significantly differ between the two groups. Neither death nor perforation occurred in either group.

PEP occurred in 27 of the 320 patients (8.4\%): 8 patients in the GWC group (5\%; 4 mild, 3 moderate, 1 severe) and 19 patients in the CC group (11.9\%; 14 mild, 4 moderate, 1 severe) $(P=.027)$. In the GWC group, PEP was significantly associated with the following: number of pancreatic cannulations during primary $\mathrm{CBD}$ cannulation attempts $(P=.001)$; number of attempts to achieve primary biliary cannulation $(P=.006)$; number of pancreatic opacifications at crossover $(P<.001)$; and number of attempts to achieve CBD cannulation at crossover $(P=.001)$, precut $(P<.001)$, and pancreatic sphincterotomy $(P<.001)$. 
On univariate analysis, risk factors for PEP in the GWC group were the following: number of pancreatic cannulations at the primary attempt $(P<.001)$, number of CBD cannulation attempts $(P=.02)$, number of pancreatic opacifications at crossover $(P=.001)$, and number of attempts to achieve CBD cannulation at crossover $(P=.005)$ and precut $(P=.001)$. Multivariate analysis identified as an independent risk factor for PEP only the number of pancreatic cannulations at the primary attempt $(P=.003)$.

In the CC group, PEP was related to pancreatic sphincterotomy $(P=.036)$ and endoscopic nasobiliary drainage $(P=.005)$. On univariate analysis, risk factors for PEP in the CC group were pancreatic sphincterotomy $(P=.021)$ and nasobiliary drainage $(P=.002)$. On multivariate analysis, pancreatic sphincterotomy and the placement of a nasobiliary drain were confirmed as independent risk factors for PEP $(P=.015$ and $P=.002$, respectively $)$.

Post-ERCP hyperamylasemia, assessed at 24 hours after the procedure, occurred in $46.9 \%$ (75/160) of patients in the GWC group and $56.2 \%(90 / 160)$ of patients in the CC group $(P=$ n. s.).

Post-ERCP hyperamylasemia in the GWC group showed an association with previous acute pancreatitis or recurrent episodes of acute pancreatitis $(P=.031)$, cholangitis $(P=.001)$, number of unintentional PD cannulations at primary attempts $(P=.007)$, number of attempts to achieve CBD cannulation $(P<.001)$, number of pancreatic opacifications at crossover $(P=.04)$, number of attempts to achieve CBD cannulation at crossover $(P=.019)$ and precut $(P=.027)$, and nasobiliary drainage $(P=.004)$.

Hyperamylasemia in the $\mathrm{CC}$ group was related to previous acute or recurrent pancreatitis $(P=.002)$, a diameter of the CBD of $10 \mathrm{~mm}$ or less $(P=.021)$, number of pancreatic ductal opacifications at primary attempts $(P=.02)$, and number of attempts to achieve primary CBD cannulation $(P=.002)$.

Post-ERCP bleeding occurred in $1.6 \%(5 / 320)$ of patients ( 2 patients in the GWC group and 3 patients in the CC group; $P=n$.s.). Hemostasis was ensured with either endoscopic or conservative management. Bleeding in the GWC group was related to previous acute or recurrent pancreatitis $(P=.009)$, number of attempts to achieve primary CBD cannulation $(P=.022)$, and number of biliary cannulation attempts at crossover $(P=.002)$, the last being the only risk factor for bleeding on univariate analysis $(P=.027)$. Bleeding in the CC group was significantly associated with cholangitis $(P=.002)$. On univariate analysis, cholangitis appeared to be the only risk factor for bleeding $(P=.022)$.

Mallory-Weiss syndrome developed in one patient in the GWC group after ERCP that required endoscopic hemostasis with clips.

\section{Discussion}

$\nabla$

To the best of our knowledge, this is the first prospective study assessing the use of the Fusion LoopTip Wire Guide with atraumatic tip for CBD cannulation and the first randomized controlled trial comparing the GWC technique and the CC technique for the prevention of PEP in the setting of high risk patients.

Patient-related and procedure-specific factors known to increase the risk for PEP include female gender, young age ( $<40$ years), suspected sphincter of Oddi dysfunction, difficult cannulation with prolonged cannulation attempts, and contrast injection of the PD [4]. Moreover, Nakai et al. recently showed that guidewire insertion into the PD is a risk factor for PEP [7]. Numerous efforts have been made to identify pharmacological agents with the potential to prevent PEP; however, the evidence has been inconclusive $[8,9]$.
Various procedural techniques intended to minimize the risk for the development of PEP have been assessed, but only GWC and prophylactic PD stenting have proved somewhat effective [10]. Omuta et al. found that a J-shaped guidewire facilitated selective biliary cannulation [11], but Tsuchiya et al. demonstrated, in a prospective multicenter controlled study, that the J-tip guidewire was not significantly different from other guidewires in regard to rates of CBD cannulation [12]. Most published studies comparing the effectiveness of GWC and CC have shown divergent results for the two techniques in both preventing PEP and increasing the biliary cannulation rate [13].

Vandervoort et al. [14] reported a higher rate of PEP, up to $10.2 \%$, associated with the GWC technique (overall PEP complication rate of $7.2 \%$ ); however, in this study, the patients undergoing GWC were probably a subgroup who had failed CC and were therefore at higher risk for the development of pancreatitis. On the other hand, in the study by Artifon et al. [15], GWC was associated with a significantly lower likelihood of PEP (adjusted odds ratio [OR] $0.43,95 \% \mathrm{CI} 0.21-0.89, P=.02$ ); however, about $11 \%$ of patients had suspected sphincter of Oddi dysfunction, which independently increases the risk for PEP.

Recently, Sasahira et al. showed that during wire-guided cannulation, conversion to a double-guidewire technique when the wire cannulated the PD at first attempt neither facilitated selective CBD cannulation nor decreased PEP incidence compared with repeated use of a single-wire technique [16]. This finding is consistent with the study by Lee et al. [17], in which GWC was associated with a lower rate of PEP than CC, but multivariate analysis showed that GWC does not prevent PEP in patients with suspected sphincter of Oddi dysfunction and unintentional PD guidewire cannulation.

In a cohort of 400 patients observed by Lella et al. [18], equally randomized to bile duct cannulation with a 0.035 -in soft-tipped Teflon guidewire or to traditional methods of cannulation, none of the patients in the GWC group developed pancreatitis, whereas the incidence of PEP in the CC group was $4 \%$.

Nambu et al. [19] found a lower incidence of PEP with the GWC method than with the contrast injection technique, but the rate of successful CBD cannulation was comparable between the two groups.

In the study by Katsinelos et al. [20], a total of 332 patients were randomly assigned to cannulation with a standard catheter $(\mathrm{n}=$ $165)$ or a hydrophilic guidewire $(n=167)$; the primary success rate of selective CBD cannulation was higher in the GWC group (81.4\%) than in the standard catheter group (53.9\%; $P<.001)$, but the frequencies of post-interventional pancreatitis and hemorrhage did not differ.

These results are also in accordance with the study of Bailey et al. [21], who demonstrated that the GWC technique improved the primary success rate for CBD cannulation, although it did not reduce the incidence of PEP compared with the CC technique.

Finally, in the latest prospective multicenter randomized controlled crossover trial to address this issue, Kobayashi et al. [22] showed that the GWC technique does not reduce the risk for PEP and does not improve the success rate of selective bile duct cannulation.

In our study, as in previous studies [15 - 19], the incidence of PEP was significantly lower with the GWC technique than with the CC technique. The overall rate of PEP was $8.4 \%$ (27/320 patients), slightly higher than that in previously published data (mean rate of 5.5\%) [15-22]. A possible explanation is that our study included only patients at high risk for PEP. We allowed five chances of 
Table 3 Summary of studies comparing contrast cannulation (CC) with standard guidewire cannulation (GWC) of the common bile duct, reported in the discussion.

\begin{tabular}{|c|c|c|c|c|c|}
\hline & Patients & $\begin{array}{l}\text { Post-ERCP } \\
\text { pancreatitis, \% }\end{array}$ & Primary cannulation, \% & Precut, \% & Complications, \% \\
\hline \multicolumn{6}{|l|}{ Non-crossover trials } \\
\hline \multirow[t]{2}{*}{ Lella et al. [18] } & GWC: 200 & GWC: $0(0 / 200)$ & GWC: $98.5(197 / 200)$ & - & - \\
\hline & CC: 200 & CC: $4(8 / 200)$ & CC: $97.5(195 / 200)$ & - & - \\
\hline \multirow[t]{2}{*}{ Artifon et al. [15] } & GWC: 150 & GWC: $3.3(5 / 150)$ & GWC: $88(132 / 150)$ & GWC: $8,7(13 / 150)$ & GWC: $5.3(8 / 150)$ \\
\hline & CC: 150 & CC: $12(18 / 150)$ & CC: $72(108 / 150)$ & CC: $22(33 / 150)$ & CC: $6(9 / 150)$ \\
\hline \multirow[t]{2}{*}{ Lee et al. [17] } & GWC: 150 & GWC: $2.0(3 / 150)$ & GWC: $80(120 / 150)$ & GWC: $18(28 / 150)$ & - \\
\hline & CC: 150 & CC: $11.3(17 / 150)$ & CC: $74(111 / 150)$ & CC: $24(36 / 150)$ & - \\
\hline \multicolumn{6}{|l|}{ Crossover trials } \\
\hline \multirow[t]{2}{*}{ Bailey et al. [21] } & GWC: 215 & GWC: 7.4 (16/215) & GWC: $77.7(167 / 215)$ & GWC: $11.6(25 / 215)$ & - \\
\hline & CC: 215 & CC: $6.0(13 / 215)$ & CC: $73(156 / 215)$ & CC: $13.5(29 / 215)$ & - \\
\hline \multirow[t]{2}{*}{ Katsinelos et al. [20] } & GWC: 167 & GWC: $5.4(9 / 167)$ & GWC: $81.4(136 / 167)$ & GWC: 15 (25/167) & GWC: $3.0(5 / 167)$ \\
\hline & CC: 165 & CC: $7.9(13 / 165)$ & CC: $53.9(89 / 165)$ & CC: $18.8(31 / 165)$ & CC: $3.0(5 / 165)$ \\
\hline \multirow[t]{2}{*}{ Nambu et al. [19] } & GWC: 86 & GWC: $2.3(2 / 86)$ & GWC: $77.9(67 / 86)$ & GWC: $3.5(3 / 86)$ & GWC: $3.5(3 / 86)$ \\
\hline & CC: 86 & CC: $5.8(5 / 86)$ & CC: 72.1 (62/86) & CC: $4.6(4 / 86)$ & CC: $7.0(6 / 86)$ \\
\hline \multirow[t]{2}{*}{ Kawakami et al. [10] } & GWC: 199 & GWC: 4.0 (8/199) & GWC: 71.4 (142/199) & GWC: 4 (8/199) & GWC: 2 (4/199) \\
\hline & CC: 201 & CC: $3.0(6 / 201)$ & CC: $69.6(140 / 201)$ & CC: $3(6 / 201)$ & - \\
\hline \multirow[t]{2}{*}{ Kobayashi et al. [22] } & GWC: 163 & GWC: $6.1(10 / 163)$ & GWC: $83(136 / 163)$ & GWC: 3 (5/163) & GWC: $1.2(2 / 163)$ \\
\hline & CC: 159 & CC: $6.3(10 / 159)$ & CC: 87 (138/159) & CC: 4 (6/159) & CC: $3(3 / 159)$ \\
\hline
\end{tabular}

ERCP, endoscopic retrograde cholangiopancreatography

main PD cannulation for the GWC group and three chances for the CC group only for the study protocol. However, despite our having selected only high risk patients, the rate of PEP in the GWC group was $5 \%$, similar to that previously reported in the literature (mean rate of $4.7 \%$ ) [15-22].

These results suggest that the design of this new guidewire with an atraumatic loop tip may reduce injury to the pancreatic and biliary ducts during cannulation attempts. The loop-shaped tip may facilitate passage through the epithelial folds of the intraduodenal biliary segments, reducing possible trauma, whereas standard guidewire tips may become stuck in the epithelial folds or cause flexion of the intraduodenal biliary segments, increasing the risk for papillary trauma during attempts at CBD cannulation. Trauma to the papilla resulting from repeated attempts at cannulation and/or increased pressure within the PD due to contrast injection are major risk factors for PEP; however, other factors have also been shown to increase the risk for PEP, include female sex, younger age, sphincter of Oddi dysfunction, and pancreatic interventions, such as pancreatic sphincterotomy [6,23].

Several mechanisms have been postulated to explain how PEP is prevented with the GWC technique. They include facilitated selective biliary cannulation, limited papillary trauma, and minimized inadvertent injection of contrast into the PD or the papilla itself, which reduce potential mechanical, chemical, and hydrostatic injury to the pancreas in comparison with the CC technique. However, inadvertent guidewire insertion into the PD may result in injury to the papilla or the PD, increasing the likelihood of PEP.

This finding was evident in our study; however, multivariate analysis identified only the number of pancreatic cannulations at the primary attempt as an independent risk factor for PEP in the GWC group. Parameters inducing trauma to the papilla, such as the number of attempts at CBD cannulation, number of attempts at biliary cannulation at crossover and precut, and number of pancreatic opacifications with use of the $\mathrm{CC}$ technique at crossover, were identified as risk factors for PEP only on univariate analysis in the GWC group.
In the $\mathrm{CC}$ group, in accordance with the findings of previous studies $[6,23]$, on multivariate analysis only pancreatic sphincterotomy and the placement of a nasobiliary drain were found to be independent risks factors for PEP.

As in the study of Nambu et al. [19], in our series the GWC technique showed no significant advantage over the CC technique with regard to the success rate of biliary cannulation. This finding might be related to the fact that only experienced endoscopists with similar skill levels for both GWC and CC were involved in the study. Risks associated with the GWC technique include perforation of the bile duct, $\mathrm{PD}$, and duodenum by the guidewire.

The studies comparing CC with standard GWC of the common bile duct are summarized in $\bullet$ Table 3 .

In our study, complications consisted of 5 cases of post-sphincterotomy bleeding (1.6\%) and 1 case of Mallory-Weiss syndrome. No perforation or mortality occurred, and as in previous reports $[14,15,20,23]$, the overall complication rates did not differ significantly between the two groups. A future randomized controlled trial comparing the loop-tip wire and a standard wire is need.

In conclusion, in our study of a high risk cohort, the use of a new guidewire with an atraumatic loop tip for biliary cannulation resulted in a significantly lower incidence of PEP than did the CC method. However, the rate of successful selective biliary cannulation and the incidence of complications were comparable between the two groups.

Competing interests: There are no financial arrangements or commercial associations (e.g., equity ownership or interest, consultancy, patent and licensing agreement, or institutional and corporate associations) that might be a conflict of interest in relation to the manuscript submitted.

\section{Institutions}

${ }^{1}$ Gastrointestinal Endoscopy Unit, San Paolo University Hospital,

University of Milan, Milan, Italy

2 Unit of Gastroenterology and Digestive Endoscopy, ARNAS Garibaldi,

Catania, Italy

${ }^{3}$ Digestive Endoscopy Unit, Hospital of Busto Arsizio, Busto Arsizio,

Varese, Italy 
${ }^{4}$ Gastroenterology Department, University of Milan, IRCCS Fondazione Policlinico, Milan, Italy

${ }^{5}$ Unit of Digestive Endoscopy, University Hospital L. Sacco, Milan, Italy

${ }^{6}$ Gastroenterology Unit, Valduce Hospital, Como, Italy

${ }^{7}$ Division of Gastroenterology and Gastrointestinal Endoscopy, Vita-Salute San Raffaele University, Scientific Institute San Raffaele, Milan, Italy

${ }^{8}$ Department of Statistics, University of Messina, Messina, Italy

\section{References}

1 Cotton PB, Lehman G, Vennes J et al. Endoscopic sphincterotomy complications and their management: an attempt at consensus. Gastrointest Endosc 1991; 37: 383 - 393

2 Masci E, Toti G, Mariani A et al. Complications of diagnostic and therapeutic ERCP: a prospective multicenter study. Am J Gastroenterol 2001; 96: 417-423

3 Freeman ML, Nelson DB, Sherman $S$ et al. Complications of endoscopic biliary sphincterotomy. N Engl J Med 1996; 335: 909-918

4 Masci E, Mariani A, Curioni $S$ et al. Risk factors for pancreatitis after endoscopic retrograde cholangiopancreatography: a meta-analysis. Endoscopy 2003; 35: 830-834

5 Freeman ML, Guda NM. ERCP cannulation: a review of reported techniques. Gastrointest Endosc 2005; 61: 112 - 125

6 Cotton PB, Garrow DA, Gallagher J et al. Risk factors for complications after ERCP: a multivariate analysis of 11,497 procedures over 12 years. Gastrointest Endosc 2009; 70: 80-88

7 Nakai $Y$, Isayama $\mathrm{H}$, Sasahira $\mathrm{N}$ et al. Risk factors for post-ERCP pancreatitis in wire-guided cannulation for therapeutic biliary ERCP. Gastrointest Endosc 2015; 81: 119-126

8 Woods KE, Willingham FF. Endoscopic retrograde cholangiopancreatography associated pancreatitis: a 15 year review. World J Gastrointest Endosc 2010; 2: 165-178

9 Enns R, Lee S. Preventing post-ERCP pancreatitis. Gastrointest Endosc 2013; 78: $851-853$

10 Kawakami H, Maguchi H, Mukai T et al. A multicentric prospective, randomized study of selective bile duct cannulation performed by multiple endoscopists: the BIDMEN study. Gastrointest Endosc 2012; 75: $362-372$

11 Omuta S, Maetani I, Shigoka H et al. Newly designed J-shaped tip guidewire: a preliminary feasibility study in wire-guided cannulation. World J Gastroenterol 2013; 19: 4531 -4536
12 Tsuchiya T, Itoi T, Maetani I et al. Effectiveness of the J-tip guidewire for selective biliary cannulation compared to conventional guidewires (the JANGLE study). Dig Dis Sci Apr 23. [Epub ahead of print]

13 Mariani A, Giussani A, Di Leo M et al. Guidewire biliary cannulation does not reduce post-ERCP pancreatitis, compared with the contrast injection technique in low-risk and high-risk patients. Gastrointest Endosc 2012; 75: 339-346

14 Vandervoort J, Soetikno RM, Tham TC et al. Risk factors for complications after performance of ERCP. Gastrointest Endosc 2002; 56: 652656

15 Artifon EL, Sakai P, Cunha JE et al. Guidewire cannulation reduces risk of post-ERCP pancreatitis and facilitates bile duct cannulation. Am J Gastroenterol 2007; 102: 2147-2153

16 Sasahira $N$, Kawakami $H$, Isayama $H$ et al. Early use of double-guidewire technique to facilitate selective bile duct cannulation: the multicenter randomized controlled EDUCATION trial. Endoscopy 2015; 47: $421-429$

17 Lee TH, Park do H, Park JY et al. Can wire-guided cannulation prevent post-ERCP pancreatitis? A prospective randomized trial. Gastrointest Endosc 2009; 60: 444-449

18 Lella F, Bagnolo F, Colombo $E$ et al. A simple way of avoiding post-ERCP pancreatitis. Gastrointest Endosc 2004; 59: 830-834

19 Nambu T, Ukita T, Shigoka $\mathrm{H}$ et al. Wire-guided selective cannulation of the bile duct with a sphincterotome: a prospective randomized comparative study with the standard method. Scand J Gastroenterol 2011; 46: 109-115

20 Katsinelos P, Paroutoglou G, Kountouras J et al. A comparative study of standard ERCP catheter and hydrophilic guide wire in the selective cannulation of the common bile duct. Endoscopy 2008; 40: 302-307

21 Bailey AA, Bourke MJ, Williams SJ et al. A prospective randomized trial of cannulation techniques in ERCP: effect on technical success and post-ERCP pancreatitis. Endoscopy 2008; 40: 296-301

22 Kobayashi G, Fujita N, Imaizumi K et al. Wire-guided biliary cannulation technique does not reduce the risk of post-ERCP pancreatitis: multicenter randomized controlled trial. Dig Endosc 2013; 25: 295 302

23 Freeman ML, Di Sario JA, Nelson DB et al. Risk factors for post-ERCP pancreatitis: a prospective, multicenter study. Gastrointest Endosc 2001; 54: $425-434$ 